\title{
Surveillance System for Multiple Moving Objects
}

\author{
Daisuke Matsuka $^{* a)}$ Member, Masahiro Mimura** Non-member
}

\begin{abstract}
To prepare for terrorist and/or criminal attacks using unmanned aerial vehicles (UAVs), we developed a Galvano Camera System. This system, which detects suspicious UAVs, is based on the high-speed and precise positioning technology of galvanometer scanners. The proposed system consists of galvanometer scanners, a zoom lens, a CMOS camera, an imaging processor, and a monitor. It is used with other position measurement sensors such as RADAR. The system changes the deflection angles of the galvano-mirrors, which are attached to the tip of the swaying shaft based on the location of the target provided by other sensors. Three targets are observed using proposed imaging process and changing angular process, alternately, to acquire multiple images that include the targets. The imaging processor makes three movies of each target, the monitor divides the screen, and displays these three movies on the same screen. The proposed system can simultaneously observe multiple moving objects, using the high response feature of the galvanometer scanners, and display objects on a single monitor. In addition, we have proved the effectiveness of the system through field tests.
\end{abstract}

Keywords: multiple moving objects surveillance, galvanometer scanner, visual servoing, UAV

\section{Introduction}

With the recent developments in unmanned aerial vehicles (UAV) and peripheral technologies, UAVs are increasingly being applied for commercial uses ${ }^{(1)-(3)}$. This is expected to expand to various applications, such as 3D surveying of construction sites, making use of the high maneuverability of UAVs.

However, reports of incidents involving UAVs have attracted public attention. For example, a multicopter was found on the roof of the Japanese prime minister's office ${ }^{(4)}$, airport runways in the US and Europe have been closed down due to sightings of UAVs nearby ${ }^{(5)}$, and an environmental group crashed a drone into a nuclear facility ${ }^{(6)}$. These incidents demonstrate that readily available commercial UAVs pose a security threat, so there is an urgent need to establish ways to deal with suspicious UAVs.

There are two stages in dealing with suspicious UAVs: first they are tracked and photographed (monitoring stage), and then they are removed from the airspace. For the monitoring stage, systems have been proposed which combine widearea measurement equipment such as microphone arrays or radar with pan-tilt-zoom (PTZ) cameras, as commonly used in surveillance applications ${ }^{(7)(8)}$. In these systems, when multiple suspected UAVs are detected by the wide-area measurement equipment, the operator visually identifies these one by one from the camera images, to make up for the poor detection accuracy of wide-area measurement equipment.

\footnotetext{
a) Correspondence to: Daisuke Matsuka. E-mail: daisuke.matsu ka.ea@hitachi.com

${ }^{*}$ Research \& Development Group, Hitachi Ltd.

832-2, Horiguchi, Hitachinaka, Ibaraki 312-0034, Japan

${ }^{* *}$ Defence Systems Business Unit, Hitachi, Ltd.

292, Yoshida-cho, Totsuka-ku, Yokohama, Kanagawa 244-0817, Japan
}

A PTZ camera consists of a camera equipped with a zoom lens mounted on an electrical platform that can turn and tilt the optical axis up and down, so it has a wide range of motion. However, it is difficult to change the optical axis of the camera at high speed because the part driven by the electrical platform is heavy. Therefore, if multiple suspected UAVs are detected by the wide-area measurement equipment, it takes time to change the optical axis of the camera, which could delay detection of suspicious UAVs. Also, while one UAV is being tracked, it cannot check a new target detected by the wide-area measurement equipment. It is therefore necessary to install multiple PTZ cameras, based on the size of the area to be monitored and the estimated number of suspected UAVs to be detected by the wide-area measurement equipment.

Meanwhile, there have also been proposals to use fixed cameras to photograph an object in any position by rotating mirrors to change the reflection angle ${ }^{(9)(10)}$. Another proposal $^{(12)}$ suggests a method of photographing objects moving at high speed using a galvanometer scanner ${ }^{(11)}$, a means of rotating mirrors which combines high responsiveness with positioning performance.

In this report, we propose a camera system using galvanometer scanners to simultaneously track and photograph multiple moving objects. The proposed system enables continuous imaging with the optical axis in multiple directions, by timing the optical axis change operation and imaging operation such that they do not overlap. The mirrors are completely stationary during imaging, which avoids blurring of the image. By identifying the position of moving objects in the image obtained and reflecting the result in the next mirror rotation angle, this system can track multiple moving objects. Therefore, our proposed system allows clear, automatic tracking of multiple objects moving at high speeds, such as UAVs. We verified the effectiveness of the proposed system in outdoor testing using a prototype. 


\section{System for Simultaneous Monitoring of Multi- ple Targets}

2.1 System Configuration Figure 1 shows our proposed system for simultaneous monitoring of multiple targets. In the proposed system, the "galvano camera" consists of the camera, the galvano unit which changes the optical axis of the camera, and the zoom lens positioned between the camera and galvano unit. The "galvano camera system" consists of the galvano camera, the controller which controls the camera, galvano unit and zoom lens, the image processor, which creates a moving image from the still images and derives the position of the objects in the image, and the image display monitor.

The galvano unit used in this system consists of a pair of galvanometer scanners with reflection mirrors on the ends and a drive amplifier to drive the galvanometer scanners. The two galvanometer scanners are arranged such that their scanning directions are at right angles to each other. In the galvano unit set up as shown in Fig. 2, the rotation angle of reflection mirror $X$ (the one closest to the zoom lens) is the horizonal deflection angle (turning angle), while the other reflection mirror $\mathrm{Y}$ has a vertical deflection angle (elevation angle). The galvanometer scanners have a structure called a direct drive system, in which the load is directly attached to the rotating shaft. This structure has high mechanical rigidity, providing high responsiveness. Therefore, as a means of

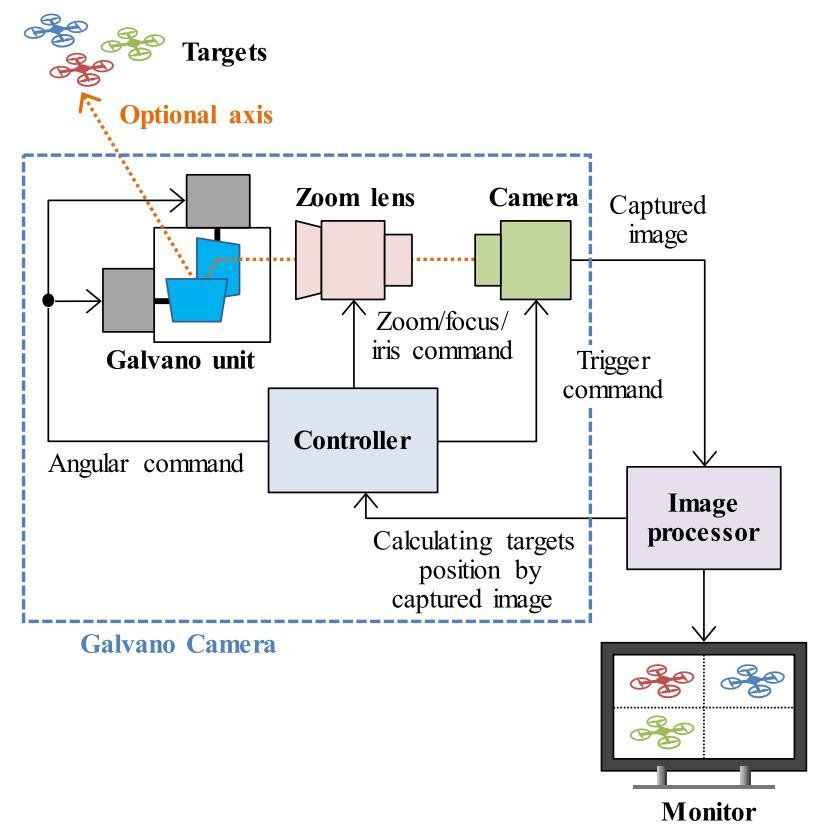

Fig. 1. Configuration of Galvano Camera System

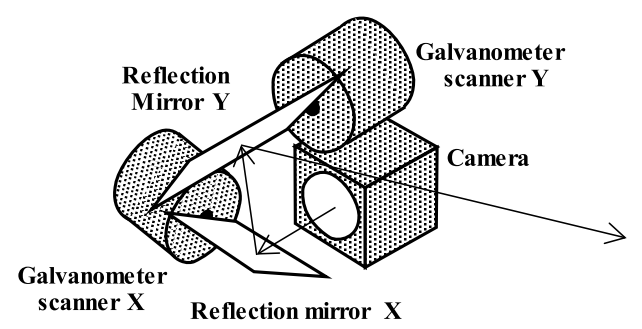

Fig. 2. Diagram of Galvano Camera changing the optical axis of a camera, this system enables faster and more precise changing of the optical axis than a camera unit with the usual PTZ mechanism. Because the heavy parts, such as the camera and zoom lens, are fixed and only the lightweight reflection mirrors move, the drive motor and mechanism are smaller than those of a PTZ camera. This provides advantages such as reduced vibration from the driving reaction force.

However, when the reflection mirrors are driven by galvanometer scanners, there is a reciprocal relationship between the size of the reflecting surface and the responsiveness of the reflection mirrors. If large reflection mirrors are selected to secure a wide angle of view, it takes longer to change the optical axis, increasing the length of the image update cycle and reducing the responsiveness. Conversely, if the reflection mirrors are too small, the angle of view becomes narrow, so it may not be possible to obtain the required imaging area. Therefore, the performance of the system is determined by the specifications of the reflection mirrors.

The camera used is selected based on the estimated maximum number of targets and the desired image update cycle. For example, to simultaneously update three targets at $10 \mathrm{fps}$, the frame rate of the camera itself must be at least $30 \mathrm{fps}$. The number of pixels and sensitivity are selected according to the environment where the system is to be used.

2.2 Calculation of Rotation Angle of Reflection Mirrors This section explains how to calculate the rotation angle of the reflection mirrors in the controller of this system. As shown in Fig. 3, the galvano camera is assumed to be at the origin position, and the subject being photographed is at $P(x, y, z)$. When the optical axis is facing toward the subject, the rotation angles $\varphi$ (turning angle) and $\theta$ (elevation angle) of each galvanometer scanner are given as

$$
\begin{aligned}
& \theta=\arccos \left(\frac{z}{\sqrt{x^{2}+y^{2}+z^{2}}}\right) \\
& \varphi=\operatorname{sgn}(y) \arccos \left(\frac{x}{\sqrt{x^{2}+y^{2}}}\right) .
\end{aligned}
$$

where

$$
\operatorname{sgn}(y)=\left\{\begin{array}{ll}
1 & (y \geq 0) \\
-1 & (y<0)
\end{array} .\right.
$$

By adjusting the applied voltage, such that the rotation angles of each galvanometer scanner in the control part become $\varphi$ and $\theta$, a drive current flows through the galvanometer scanners, so that the optical angle of the camera faces toward the target.

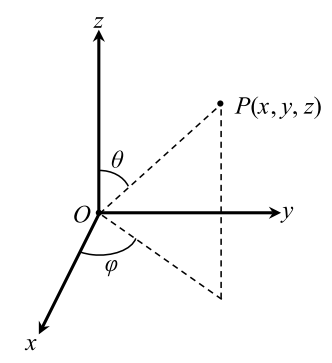

Fig. 3. Coordinate system of Galvano Camera 


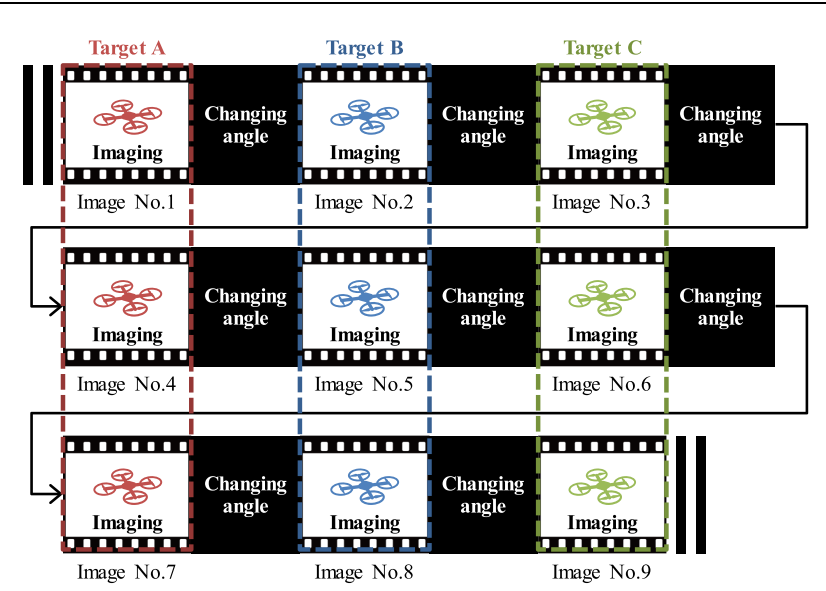

Fig. 4. Conceptual diagram of multiple target monitoring

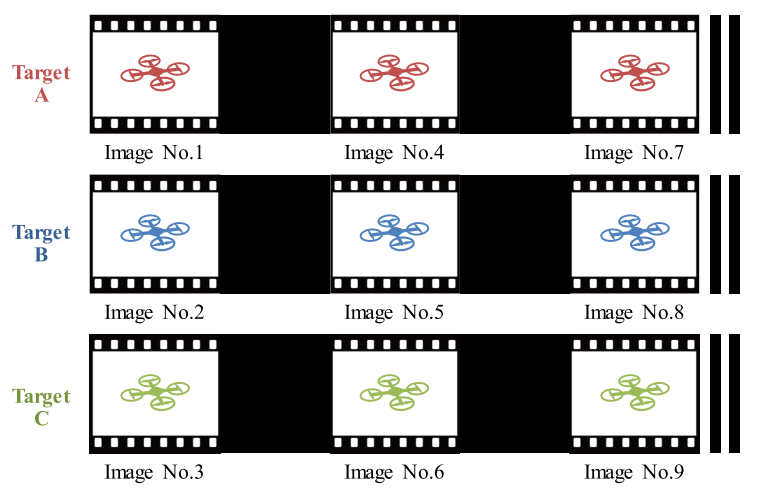

Fig. 5. Processed movies by Galvano Camera System

2.3 Simultaneous Monitoring of Multiple Targets

Using galvanometer scanners as a way to change the optical angle at high speed, the proposed system allows simultaneous monitoring of multiple targets by repeating the optical angle change operation and imaging operation alternately.

As an example, we will explain the process of monitoring three targets at the same time using this system. Figure 4 shows the images obtained by the camera and the movement of the galvano unit, as a chronological schematic diagram. When this system receives information about the position of each target from the wide-area measurement equipment, the controller calculates the rotation angles for the first subject (target A) and drives the galvanometer scanners. When the positioning operation is complete, and the optical axis of the camera is facing toward target $\mathrm{A}$, the controller sends a trigger signal to the camera, so it starts capturing images containing target $\mathrm{A}$. When it has finished capturing an image, it obtains images of targets $\mathrm{B}$ and $\mathrm{C}$ following the same procedure. In this example, it continues taking photographs in the order $\mathrm{A} \rightarrow \mathrm{B} \rightarrow \mathrm{C} \rightarrow \mathrm{A} \rightarrow \mathrm{B} \rightarrow \mathrm{C} .$.

Next, using Fig. 5, we will explain the processing in the image processor. Figure 5 shows the movie data corresponding to each subject created by the image processor of this system. The image processor takes the images of targets A, $\mathrm{B}$ and $\mathrm{C}$ from the camera and makes them into three movies (one for each target). After performing color tone correction, so that target $\mathrm{A}$ is clearly visible, the image processor stitches together the images containing target $\mathrm{A}$ in chronological order (image no. $1 \rightarrow 4 \rightarrow 7$ ) to create a movie of target $\mathrm{A}$. It carries out the same processing for targets $\mathrm{B}$ and $\mathrm{C}$ to obtain

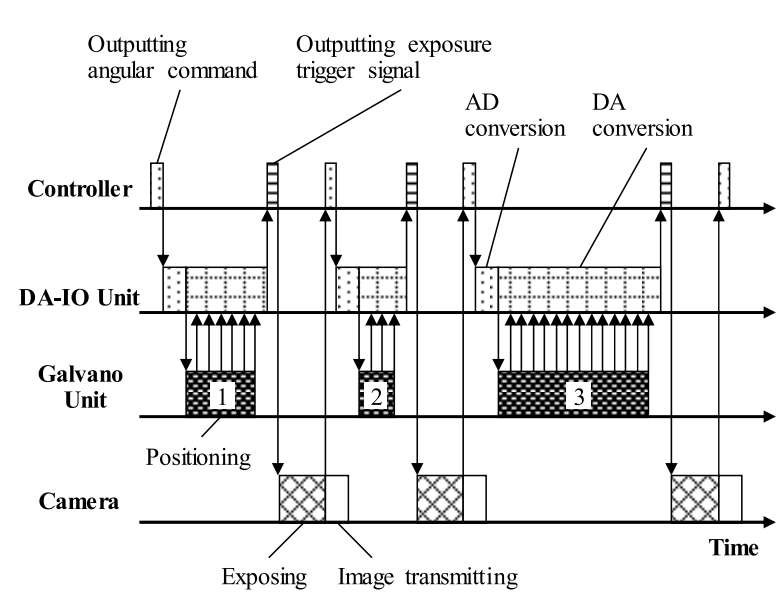

Fig. 6. Timing chart of Galvano Camera System

movie data. The update cycle for the movie data obtained is the imaging cycle $\times$ number of targets $(\mathrm{sec})$. The screen area of the monitor is split according to the number of targets, so it can display the movies for each target. This means the system displays movies of targets A, B and C simultaneously on the monitor, allowing the operator to monitor three subjects located in different directions at the same time.

2.4 Adjustment of Operation Timing When photographing multiple targets with the proposed system, the rotation angle to change the optical axis depends on the position of the subject, so the time from the start of movement to the completion of operation is not constant. In a system with a fixed imaging cycle, if the imaging cycle is too short, camera exposure may start while the optical axis is changing, resulting in a blurred image. On the other hand, if the imaging cycle is set to the maximum time required for movement, image blurring can be reduced, but the image update frequency decreases.

In the proposed system, the frame rate is maximized, and blurring is avoided by timing the optical axis change operation and imaging operation such that they do not overlap. Figure 6 shows the timing chart of the controller, digital analog input output (DA-IO) unit, galvano unit and camera in the proposed system. Figure 6 shows the operation when photographing three targets. The main processes are: digital-toanalog conversion by the DA-IO unit (approximately $1 \mathrm{~ms}$ ), analog-to-digital conversion by the DA-IO unit (shorter than $1 \mathrm{~ms}$ ), positioning operation of the galvano unit (several $\mathrm{ms}$ ), camera exposure (several $\mathrm{ms}$ ), and transmission of image data (approximately $1 \mathrm{~ms}$ ). In this example, the rotation angles differ greatly, so the operation time for positioning of the galvano unit is different for operations 1 to 3 .

First, the controller sends an angle command value to the DA-IO unit. The DA-IO unit converts the digital angle command value to an analog voltage and sends this to the galvano unit. The galvano unit performs the positioning operation based on the input voltage. The rotation angle of the reflection mirror is output as a voltage from the galvano unit, converted by the DA-IO unit, and continuously sent to the controller as the current angle. When the difference between the angle command value and the current angle is below the set value, the positioning operation is determined to be complete. Next, a signal is sent to the camera to start exposure. 
When the set exposure time has passed, the image data obtained is sent from the camera to the controller. At the same time, the controller sends the next angle command value to the DA-IO unit. If the image data transfer time is longer than the $\mathrm{AD}$ conversion processing time of the DA-IO unit, the next angle command value is sent after the transfer data processing is completed. This operation is repeated to obtain a sequence of images of multiple targets.

As explained above, this system adjusts the timing of each operation so the positioning of the galvano unit does not overlap with the exposure of the camera. This means there will be no blurring, even if the time required for the positioning of the galvano unit for each optical angle change operation differs greatly due to the positions of the multiple targets.

\section{Tracking Method for Multiple Targets}

As explained above, systems to monitor suspicious UAVs use a combination of wide-area measurement equipment and cameras. Wide-area measurement equipment can simultaneously find multiple objects in a wide monitoring area, but the position information update cycle is around 1 second. It is possible to track suspicious UAVs by driving a galvano camera based on position information obtained from wide-area measurement equipment, but there were issues with the ability to track objects moving at high speed. Utilizing the high responsiveness of the galvano camera, once a moving object is captured from the acquired image, it should be possible to improve tracking performance by detecting the direction of movement from the moving image. In this section, we explain a method of detecting and tracking moving objects from a series of images. This paper assumes operation with a fixed zoom. The magnification was set so that the subject was around 1/7.8 of the screen width in the evaluation test in Section 4. Assuming the subject to be a UAV flying at $40 \mathrm{~km} / \mathrm{h}$ in the left-right direction of the galvano camera, this size was set so that the type of UAV could be recognized from the images acquired, and it could be tracked.

The proposed method is based on the background subtraction method but is different from the conventional method in that the optical axis of the camera is changed by galvanometer scanners and that multiple targets can be tracked simultaneously. Figure 7 shows the flow chart for the image processor in the proposed method, when tracking three moving objects. The characteristic points are that it creates a background model for each of the moving objects (S02), updates the background models every time the optical axis changes (S111), and does not allow the optical axis to be changed until it has reached the set standby count $\alpha(\mathrm{S} 109) . n_{i}$ is called the standby count. The reason for specifying $\alpha$ is that if the optical axis is changed too frequently, it becomes difficult to visually recognize the image target, which is not suitable for monitoring purposes. In the evaluation test described below, $\alpha$ was set to 2 . First, the standby count and background model are initialized for each of the three moving objects ( $\mathrm{S} 01$, $\mathrm{S} 02$ ), and the subroutine is repeated until the target number of times is completed ( $\mathrm{S} 03, \mathrm{~S} 04, \mathrm{~S} 05)$. In the subroutine, the image is acquired, converted to a grayscale image, and then a $5 \times 5$ pixel median filter is applied to remove noise (S101 to S104). Next, the difference image is obtained by subtracting the filtered image from the background model, and contours

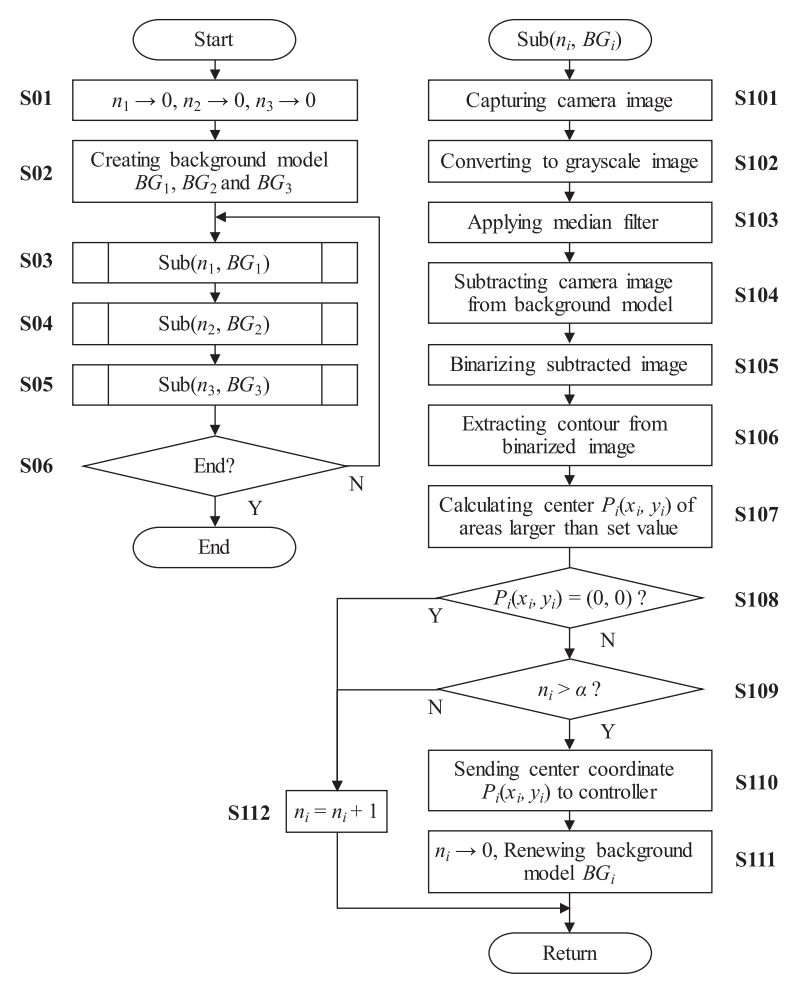

Fig. 7. Flow chart of detecting moving objects

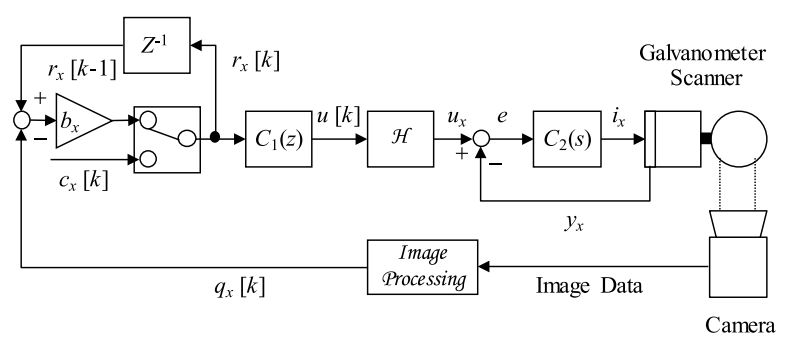

Fig. 8. Block diagram of Galvano Camera System

are extracted from the binarized image ${ }^{(13)}$ (S105, S106). For contours larger than the specified value, it calculates the center position $P_{i}(\mathrm{~S} 107)$, and if the center position $P_{i}$ of the contour is at the center of the image, it adds 1 to the standby count and returns to the main routine (S108, S112). If the center position $P_{i}$ of the contour is not at the center of the image, and the standby count is $\alpha$ or less, it adds 1 to the standby count and returns to the main routine (S109, S112). If the center position $P_{i}$ of the contour is not at the center of the image, and the standby count is greater than $\alpha$, it sends the center position of the contour to the controller, initializes the standby count and background model, and returns to the main routine (S109 to $\mathrm{S} 111$ ). As explained above, the image processor in the proposed system derives the center position of each moving object from the acquired image and sends the coordinates to the controller.

Figure 8 shows a control block diagram of galvanometer scanner $X$ in the proposed system. The control block diagram of galvanometer scanner $\mathrm{Y}$ is similar. In the diagram, $\mathrm{Z}^{-1}$ : delay operator, $b_{x}$ : command magnification, $C_{1}(z)$ : FB compensator $1, H$ : holder, $C_{2}(s)$ : FB compensator $2, r_{x}$ : angle command, $c_{x}$ : angle command value from the higher-level system, $q_{x}$ : moving target position in the image sent from the 
image processor, $u_{x}$ : operation amount, $i_{x}$ : electrical current, $y_{x}$ : detected current angle. The index $k$ indicates a value at a certain point in time, and $k-1$ indicates the value one control cycle before. $C_{1}(z)$ and $C_{2}(s)$ are the digital and analog PID compensation, respectively.

This system can switch between two modes: a mode in which the angle command value is sent from the host (referred to below as external command mode) and a mode where the angle command value is generated internally (tracking mode). External command mode is used when the moving object is not included in the acquired image. The optical axis is changed using position information about the target being monitored, obtained by the wide area measuring equipment. The outer feedback loop is not formed at this time. Tracking mode is used after a moving object has been detected in the acquired image. A new angle command value $r_{x}$ is created by comparing the position of the moving object in the image from the image processor with the angle command value one control cycle before. At this time, the angle command value is multiplied by the command magnification $b_{x}$. This is a value corresponding to changes in the zoom lens magnification. The higher the zoom lens magnification, the smaller the value of $b_{x}$, and the lower the zoom lens magnification, the larger the value of $b_{x}$.

As described above, once a moving object has been detected in the image, the proposed galvano camera system can continuously track and photograph multiple moving objects, by controlling the camera optical axis according to the amount of movement of each moving object.

It should be noted that changing the zoom magnification of a general zoom lens takes several seconds, which is significantly slower than the optical axis changing and imaging operations that are completed in a few milliseconds. Therefore, it is difficult for a galvano camera incorporating a generalpurpose zoom lens to change the magnification when tracking multiple moving objects. Changing zoom magnification at high speed according to the subject is one of the future issues to be addressed. With this system, if the moving object goes out of view during tracking, position information is obtained again from the wide-area measurement equipment, the optical axis is directed toward the subject, and the zoom magnification is simultaneously changed to a preset zoom magnification according to the distance.

\section{Evaluation Testing of the Proposed System}

4.1 Configuration of Prototype We produced a prototype to evaluate the performance of the proposed system. The parts used are listed in Table 1, the external view of the system is shown in Fig. 9, and the internal configuration of the galvano camera is shown in Fig. 10. The proposed system is made up of the galvano camera, control box and control PC. The galvano camera part consists of the galvano unit, drive amplifier, DC fan for cooling the drive amplifier, zoom lens and CMOS camera. We used a general-purpose galvano unit for laser marking (laser beam diameter: $\varnothing 30 \mathrm{~mm}$, movable range: optical angle \pm 20 degrees). The drive amplifier was cooled by forced air cooling by the cooling fan. We used a zoom lens has a variable-power ratio of 20 times. For the CMOS camera, we used a global shutter type 3.1-megapixel area scan camera with an image sensor of $1 / 1.8$ inches. The
Table 1. Parts list of Galvano Camera

\begin{tabular}{|c|c|}
\hline Part name & Specification \\
\hline Galvanometer scanner & $25 \mathrm{~A}($ peak), $8.2 \mathrm{~A}(\mathrm{RMS})$ \\
\hline Reflection mirror X & Major axis: $52.3 \mathrm{~mm}$ \\
& Miner axis: $30.0 \mathrm{~mm}$ \\
\hline Reflection mirror Y & Major axis: $62.4 \mathrm{~mm}$ \\
& Miner axis: $44.0 \mathrm{~mm}$ \\
\hline CMOS camera & $2064 \times 1544$ pixels, $120 \mathrm{fps}$ \\
\hline Zoom lens & $f=15-300 \mathrm{~mm}$ \\
\hline Control PC & Windows 10,64 bit \\
& Core i7-6700HQ, GeForce GTX 1060 \\
\hline
\end{tabular}

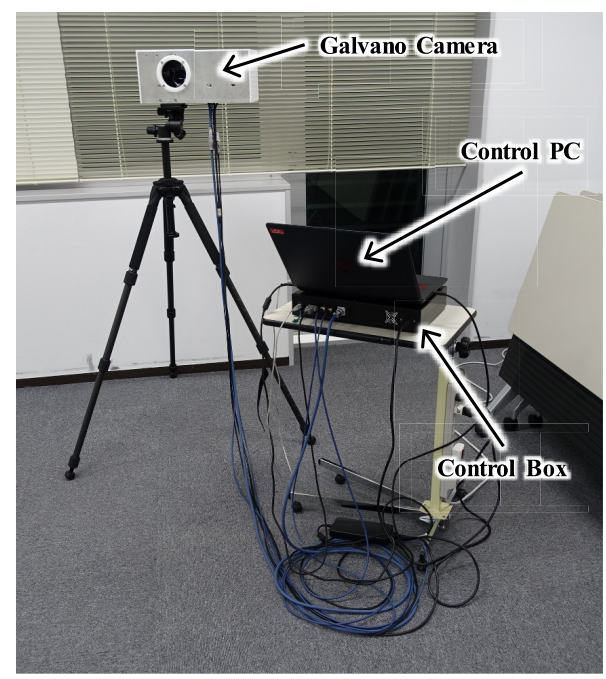

Fig. 9. External view of Galvano Camera System

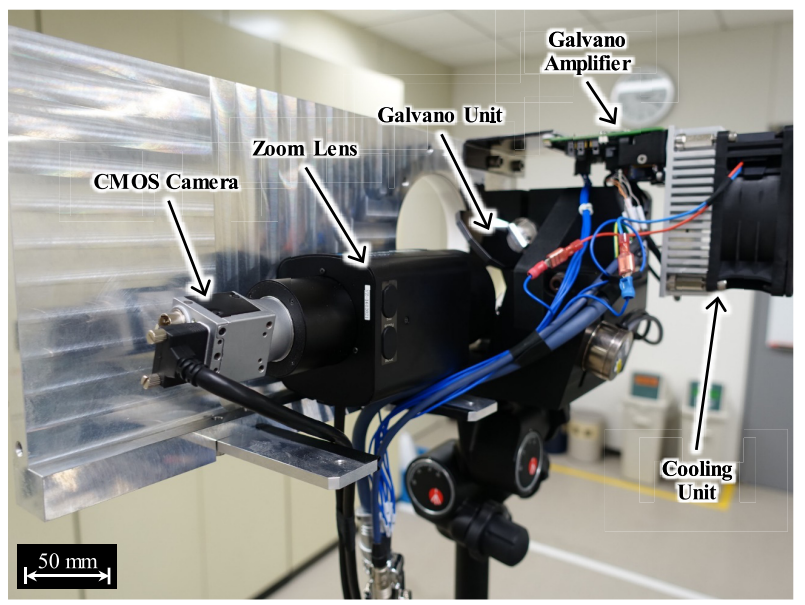

Fig. 10. Internal view of Galvano Camera

galvano camera part is lightweight, weighing $9.77 \mathrm{~kg}$, so an adult can carry the galvano camera and tripod together. We selected a laptop with a high-performance graphical processing unit (GPU) as the control PC, prioritizing portability. The system power is around $150 \mathrm{VA}$, even when the galvano unit is operating, so it can be used outdoors, powered by a storage battery.

4.2 System Configuration of Prototype Figure 11 shows the system configuration diagram of the prototype. The galvano camera control program in the diagram controls the galvano unit and camera. After performing the image 


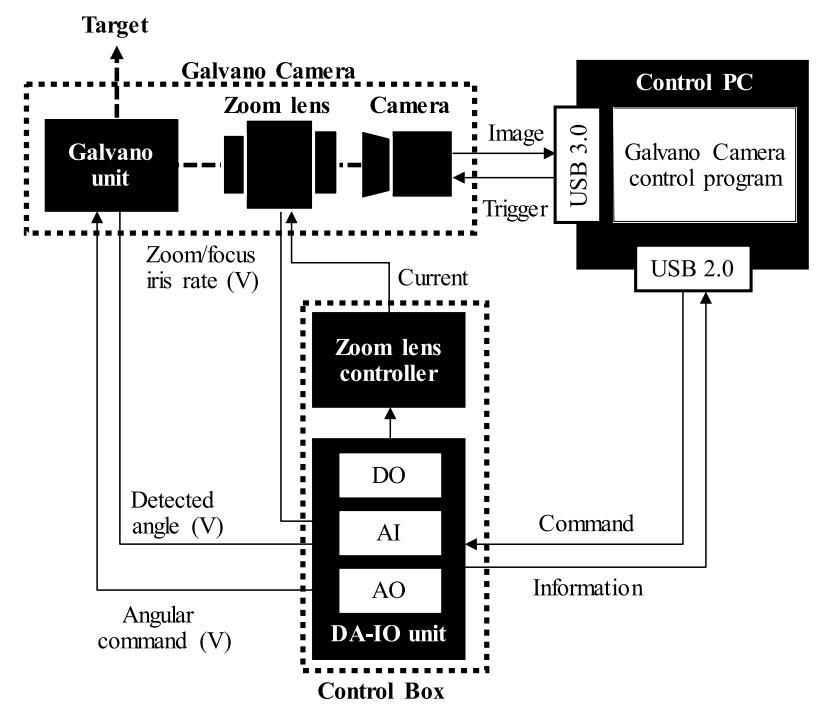

Fig. 11. External view of Galvano Camera System

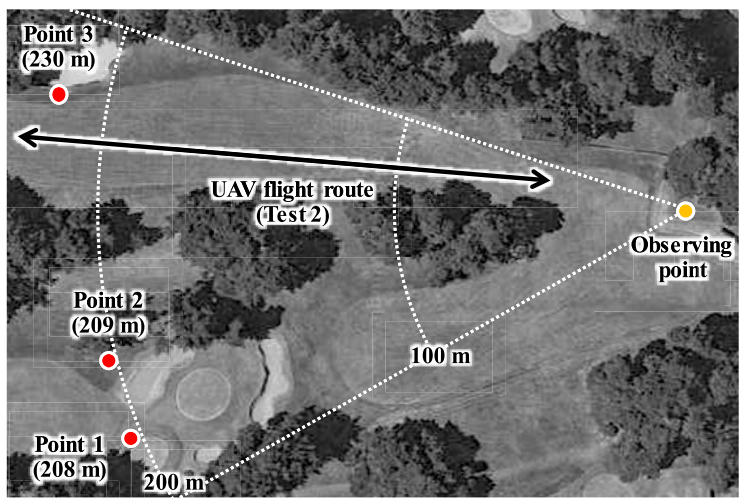

Fig. 12. Aerial photograph of test field

processing described in Sections 2 and 3, multiple targets are displayed on the monitor of the control PC. The control box contains a general-purpose microcomputer board to control the zoom lens, as well as the DA-IO unit. The control PC is connected to the DA-IO unit with a USB 2.0 cable. The exposure start signal is sent to the camera, and images are acquired via a USB 3.0 cable.

4.3 Performance Evaluation Testing Performance evaluation testing was carried out on a golf course where UAVs can be flown at high speed (Fig. 12). The galvano camera system was installed on a hill about $5 \mathrm{~m}$ higher than the surrounding area. The target to be observed was either a UAV, volunteer or balloon, depending on the test. The performance evaluation testing involved (1) Simultaneous monitoring in three directions, (2) Tracking of a single moving object, and (3) Simultaneous tacking of three moving objects. We obtained permission to fly UAVs before performing the test.

(1) Test 1: Simultaneous monitoring in three directions

First, we confirmed that the proposed system could take photographs in multiple directions at the same time. We set up a red balloon at point 1 and a yellow balloon at point 3 in Fig. 12. We got the system to display three directions at the same time, including point 2 where nothing was placed, and confirmed the frame rate and whether there was any blurring. Each target point was around $200 \mathrm{~m}$ from the observation point, and the balloons used had a diameter of around

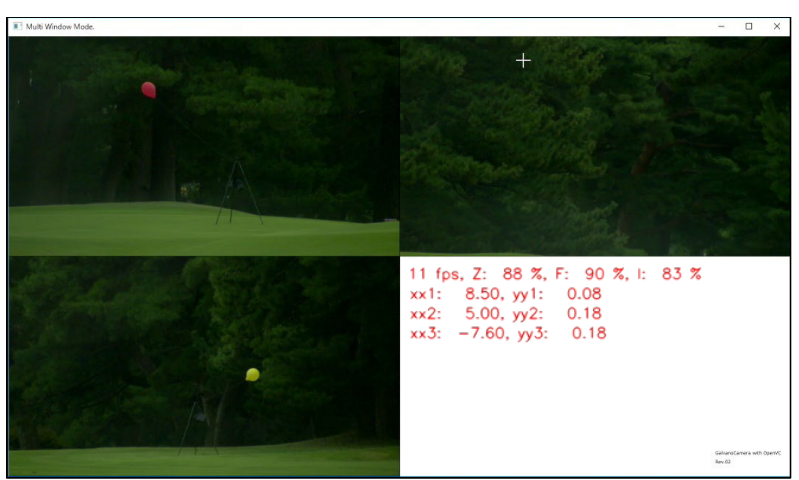

Fig. 13. Captured image of Galvano Camera System at test 1

$200 \mathrm{~mm}$. The balloons were attached to a general camera tripod using a piece of synthetic thread of length $1.5 \mathrm{~m}$ and were filled with helium gas so they floated in the air. The camera exposure time was set to $5 \mathrm{~ms}$, and images were in HD quality $(1280 \times 720$ pixels $)$.

Figure 13 shows the images extracted as still images from the movie captured in Test 1 . They were taken in the order top left (point 1) $\Rightarrow$ top right (point 2) $\Rightarrow$ bottom left (point 3 ). The numbers next to "xx1:" to "xx3:" in the bottom right of Fig. 12 indicate the turning angle (mechanical angle) of the galvanometer scanner: 8.50 degrees (17.0 degrees optical angle) for the red balloon (top left), 5.00 degrees for the top right image, and -7.60 degrees for the yellow balloon (bottom left). Each target was captured in HD quality, the length and width of each image was madehalf, the images in three directions were joined together and displayed on a single screen. The system was able to perform imaging and optical axis change operations without any blurring of the images. The frame rate was $11 \mathrm{fps}$. The results of this test indicated that the galvano camera system is capable of simultaneous monitoring in multiple directions.

(2) Test 2: Tracking of a single moving object

Next, we confirmed the effectiveness of the multiple image tracking method described in Section 3. Using a generalpurpose UAV with propeller shaft distance of $350 \mathrm{~mm}$, we confirmed the images obtained when the UAV was flown from a distance of $50 \mathrm{~m}$ to $250 \mathrm{~m}$ from the observation point, at an altitude of 10 to $20 \mathrm{~m}$. The maximum speed was about $13 \mathrm{~m} / \mathrm{s}$. The camera imaging conditions were the same as in Test 1 , and the zoom magnification was fixed.

Figure 14 shows the images extracted as a series of still images (one image per second) from the movie obtained in Test 2. The center position of the red circle, such as those shown after 0 or 2 seconds, is the center position of the moving object calculated by the image reference tracking method, which is sent to the galvano camera controller in the next optical axis change operation. In the image after 1 second, where no red circle appears, this means it is immediately after the optical axis change, when the standby count is 0 . These images confirm that the system was able to detect the UAV and capture images in HD quality while tracking the target. The frame rate was $32 \mathrm{fps}$. In the images taken after 9 seconds and 10 seconds, the UAV appears at the edge of the image. This is because the distance moved by the UAV on the screen from one optical axis change operation to the next 


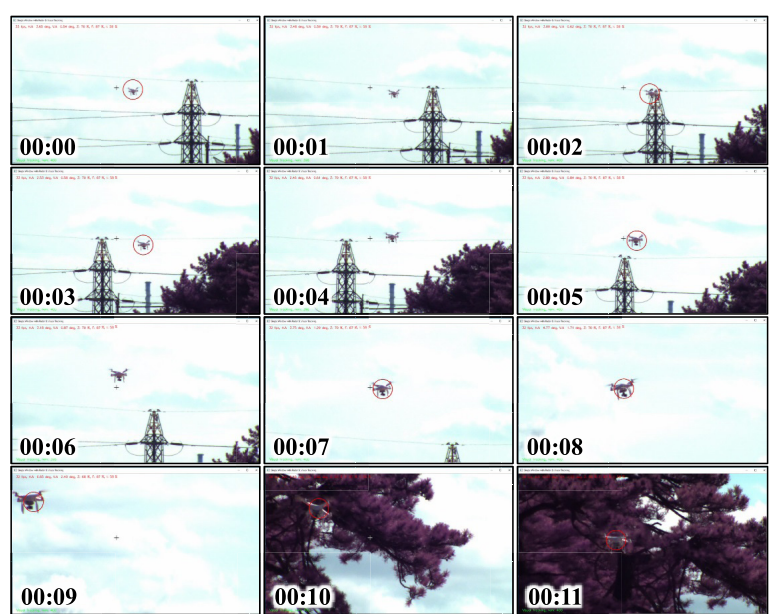

Fig. 14. Captured image of Galvano Camera System at test 2

becomes longer as the UAV approaches. In this test, it did not become impossible to track the target, but there are ways to ensure that the target always appears near the center of the image, such as reducing the standby count $\alpha$ or reducing the zoom magnification to shorten the moving distance on the screen. An appropriate response speed can be obtained by adjusting the standby count and the zoom magnification according to the assumed size and movement speed of the target to be monitored.

(3) Test 3: Simultaneous tacking of three moving objects Finally, we confirmed whether the proposed system is capable of tracking and photographing multiple moving objects simultaneously. Similar to Test 1 , we placed a red balloon at point 1 and a yellow balloon at point 3 in Fig. 12, with a volunteer moving from left to right around point 2 . We confirmed the frame rate and whether there was any blurring when tracking and photographing three moving objects with the proposed system. The camera imaging conditions were the same as in Test 1, and the zoom magnification was fixed.

Figure 15 shows the images extracted as a series of still images (one image per second) from the movie obtained in Test 3. They were taken in the order top left (point 1) $\Rightarrow$ top right (point 2) $\Rightarrow$ bottom left (point 3 ). Each target was tracked with the turning angle (mechanical angle) of the galvanometer scanner within the range of 8.62 to 8.72 degrees for the red balloon, 4.35 to 5.21 degrees for the human subject, and -7.67 to -7.72 degrees for the yellow balloon. Images were captured in HD quality while tracking each target, the length and width of each image was made half, the images in three directions were joined together and displayed on a single screen. The system was able to perform imaging and optical axis change operations without any blurring of the images. The frame rate was $9 \mathrm{fps}$. The results of this test indicated that the galvano camera system is capable of simultaneous tracking and photographing of multiple moving objects.

The findings above show that the galvano camera system is capable of monitoring and tracking multiple UAVs. The detection accuracy and tracking speed depend on the combination of camera exposure time, standby count and command magnification, so these need to be optimized when the subject
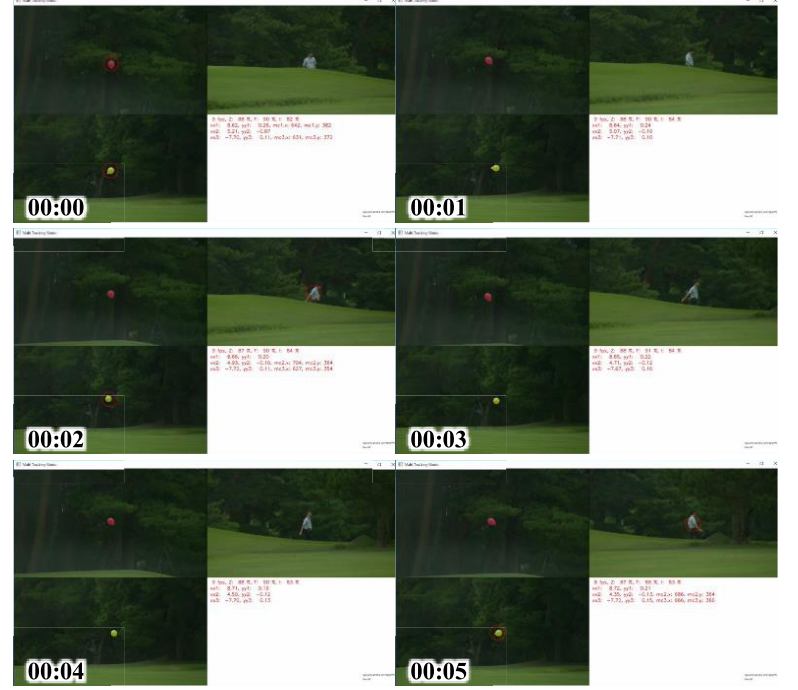

Fig. 15. Captured image of Galvano Camera System at test 3

and environmental conditions change. This is an issue to be investigated in future.

\section{Conclusion}

In this study, we proposed a camera system using galvanometer scanners in order to track and photograph multiple moving objects simultaneously. By adjusting the timing of the optical axis change operation and imaging operation, such that they do not overlap, the proposed system allows continuous imaging with the optical axis pointing in multiple directions, avoiding blurring of the images due to the optical equipment. In addition, tracking of multiple moving objects was achieved by a multiple image tracking method, whereby the angle command value of the reflecting mirrors is determined based on the acquired images for each moving object. Therefore, the proposed galvano camera system allows clear, automatic tracking of multiple objects moving at high speeds, such as UAVs. The response speed is also much faster than a conventional monitoring system using a PTZ camera, so this system is expected to provide a new monitoring method.

In this paper, we focused on changing the optical axis of the camera at high speed. We will investigate how to change the zoom magnification at high speed as an extension of this research.

\section{References}

( 1 ) Nikkei Business Publications Clean Tech Institute and TechnoAssociates, Inc.: "World Drone Overview", Nikkei Business Publications (2015)

( 2 ) H. Sasuhara and R. Kobayashi: "Survey report of drone business", Impress Corporation (2016)

( 3 ) M. Mazur, A. Wisniewski, and J. McMillan: "PwC global report on the commercial applications of drone technology", PwC Clarity from above (2016)

( 4 ) The official residence of the prime minister: "Current policy on UAV", Meeting document of related government offices for UAV (2015)

( 5 ) "Drone sighting disrupts major US airport", BBC, 2019/1/23, (https://www. bbc.com/news/technology-46968419), 2019/1/15

( 6 ) Greenpeace crashes Superman-shaped drone into French nuclear plant", Reuters, 2018/7/3, (http://www.reuters.com/article/us-france-nuclear-green peace/greenpeace-crashes-superman-shaped-drone-into-french-nuclearplant-idUSKBN1JT1JM), 2019/1/15

( 7 ) "The lunch of the drone detection system", Panasonic, Ltd., 2016/3/4, 
(https://news.panasonic.com/jp/press/data/2016/03/jn160304-1/jn160304-1. pdf)

( 8 ) "The lunch of the drone detection system that quickly finds suspicious drones", Hitachi, Ltd., 2017/10/6, http://www.hitachi.co.jp/New/cnews/ month/2017/10/1006.pdf

(9) S. Kobayashi: Japan Patent Kokai, 10-136234 (22, May, 1998)

(10) K. Yokoyama and Y. Terashima: Japan Patent Kokai, 11-308603 (05, November, 1999)

(11) D. Matsuka, K. Seki, and M. Iwasaki: "Development of High-speed and Precise Positioning for Galvanometer Scanners", JIASC2014, 2-S8-3,pp.13-18 (2014)

(12) K. Okumura, M. Ishii, E. Tatsumi, H. Oku, and M. Ishikawa: "Image Measurement of a High-speed Flying Object Using the High-speed Gaze Controller", Transactions of the Society of Instrument and Control Engineers, Vol.49, No.9, pp.855-864 (2013)

(13) S. Suzuki and K. Abe: "Topological structural analysis of digitized binary images by border following", Computer Vision, Graphics, and Image Processing, Vol.30, No.1, pp.32-46 (1985)

Daisuke Matsuka (Member) received the B.S. and M.S. degrees in in-

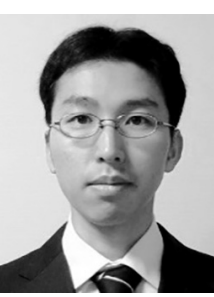

telligent mechanical engineering from Tokyo Denki University, Hatoyama, Japan, in 2003 and 2005, respectively, and the Dr.Eng. degree in computer science engineering from Nagoya Institute of Technology, Nagoya, Japan, in 2016. Since 2005, he has been with the Research and Development Group, Hitachi, Ltd., Tokyo, Japan. His current research interests include servocontrol technologies and integrated design of controllers and structures in motors for industrial equipment. Dr. Matsuka is a member of The Japan Society of Mechanical Engineers.
Masahiro Mimura (Non-member) received the Dr.Eng. degrees from

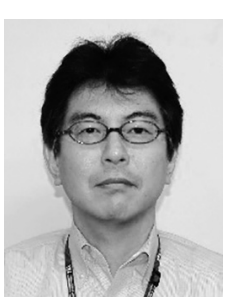
Tokyo Institute of Technology, Japan, in 1997. He is the Chief Technology Officer at the Defense Systems Business Unit, Hitachi, Ltd., Tokyo, Japan. 